\title{
Learning the function concept by exploring digital images as functions
}

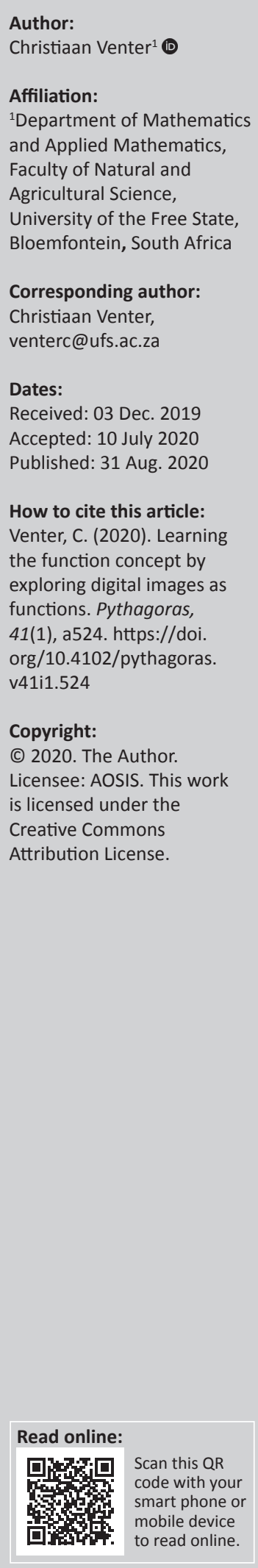

\begin{abstract}
Despite the function concept being fundamental to mathematics, an adequate understanding of this concept is often lacking. This problem is prevalent at all levels of education and is reported in many countries. This article reports on a new pedagogical strategy based on exploring photographs or digital images as functions. The objective of this study was to determine if the Image Functions Intervention (IFI) could be considered sound and viable with respect to the learning of the function concept. A two-part approach was used. The first part used the Action-Process-Object-Schema (APOS) theory as a theoretical framework for evaluating the IFI. The second part implemented the IFI with a group $(n=27)$ of undergraduate students, followed by qualitative analysis of questionnaire data in order to look for indications of participants experiencing broadened thinking with respect to the function concept. The theoretical evaluation confirmed the IFI to be sound and providing opportunities for addressing common conceptual difficulties of the function concept. The qualitative analysis provided evidence of the participants' expanded concept images. It was concluded that the designed intervention, the IFI, is sound and viable and shows promise with respect to increased understanding of the function concept.
\end{abstract}

Keywords: function concept; image functions; APOS theory; genetic decomposition; mathematics.

\section{Introduction}

That the function concept is fundamentally important to mathematics can be accepted as a commonly shared opinion. As stated by Selden and Selden in Harel and Dubinsky (1992, p. 1), 'the function concept, having evolved with mathematics, now plays a central and unifying role'. And, more recently, '[t]he concept of function is central to students' ability to describe relationships of change between variables, explain parameter changes, and interpret and analyze graphs' (Son \& Hu, 2015, p. 4). O'Shea, Breen and Jaworski (2016, p. 279) reiterate that '[f]unctions are central to present day mathematics' and elaborate that 'going beyond calculus, functions are widely used in the comparison of abstract mathematical structures'.

Despite the high value attached to an adequate understanding of functions and the function concept, a full and nuanced comprehension is not common among undergraduate students (Carlson, Jacobs, Coe, Larsen \& Hsu, 2002, p. 353) or secondary school students (Sajka, 2003, p. 229). Doorman et al. (2012, p. 1243), in working with secondary school students, confirm the difficulty in learning the function concept and in particular state that '[f]unctions have different faces, and to make students perceive these as faces of the same mathematical concept is a pedagogical challenge'. This challenge is ongoing despite more than 50 years of research, producing 'a vast literature on teaching and learning the function concept' (Dubinsky \& Wilson, 2013 , p. 84). That it remains such a challenge can partly be understood in the light of the difficulties evident in the history of the development of the function concept. The concept is said to be an epistemological obstacle (Sierpinska, 1992, p. 28) as the difficulties associated with it have been prevalent and persistent over a long time and are still commonly observed. The other reason could be attributed to what Dubinsky and Wilson (2013, p. 86) highlight as the little attention that has been paid to research that applies theoretical analyses (which is plentiful) to develop 'pedagogical strategies for helping students overcome these difficulties'. Simply put: (1) the concept of function is a difficult concept and (2) we have not been getting sufficiently practical in designing appropriate interventions, instructional treatments and didactical designs.

Some work has been done in getting practical, but, seemingly, more is needed. Ayers, Davis, Dubinsky and Lewin (1988) and Breidenbach, Dubinsky, Hawks and Nichols (1992) considered the use of simple programming environments to provide practical activities in 
creating and using functions. Tall, McGowen and DeMarois (2000) considered the use of the 'function box/machine' as a strong cognitive root to anchor the different ideas connected with the function concept. Reed (2007) researched the effect of having students actively engage with the history of the concept of function. Salgado and Trigueros (2015) based their design and activities on models and modelling.

This article reports on an intervention, the Image Functions Intervention (IFI). The IFI explores digital images considered as functions. The intervention was firstly theoretically evaluated and secondly used with a small group $(n=27)$ of undergraduate students, followed by qualitative analysis. One aspect where this intervention differed from the implementations mentioned earlier was in that participants were meant to use the intervention in a self-directed manner. This was achieved by making use of e-learning authoring software in conjunction with a learning management system, making it possible for a participant to complete the IFI at their own pace. Therefore, participants could use the IFI with no lecturer involved. The need for such a self-directed intervention arose firstly from time constraints with respect to direct contact time with students and secondly from the advantage of not needing teachers or lecturers to first become acquainted with the underlying ideas and content of the intervention.

With the function concept in mind, Akkoç and Tall (2005, p. 7) point out that even in the face of a specific design, the outcome might not be achieved. They discuss a course that was designed to make the function concept foundational and an organising principle, but instead 'many students focus on the individual properties of each representation without connecting them together'. In order to increase the probability of a design being successful, it should be based on research and theory. Salgado and Trigueros (2015) provide a good example of such a design informed by the Action-Process-Object-Schema (APOS) theory. Their design makes use of models and modelling. They first motivated their use of modelling by referring to research showing how modelling can raise motivation and interest, assist in identifying specific learning difficulties and facilitate learning and concept construction. Thereafter a genetic decomposition (defined in the theoretical framework section) was constructed from which activities could be designed.

In light of the understanding of what reasonable design implies, this article will use a theoretical evaluation guided by the APOS theory, supplemented by the literature on the learning of the function concept, to determine if it would be reasonable to expect that the IFI could improve understanding of the function concept. The following research question was formulated:

Can the IFI be considered theoretically sound and viable with respect to the learning of the function concept?

The research question was addressed in two parts.
Part 1 dealt with a theoretical evaluation of the IFI. Keeping in line with the methodology of the APOS theory, the current literature as well the researcher's own experience were incorporated to create a genetic decomposition (defined in the theoretical framework section) of the function concept (Dubinsky, 2000, p. 2; Maharaj, 2010, p. 42). This genetic decomposition (GD) proposed the appropriate mental structures at the action, process and object levels that a student could potentially need in learning the function concept. This GD was used as a guide to evaluate the soundness of the IFI. The activities of the IFI were required to link with the function-related mental structures proposed in the GD. Furthermore, it was examined if and how the IFI's activities were addressing the common conceptual difficulties (Dubinsky \& Wilson, 2013, pp. 85-86) associated with the function concept, such as 'what constitutes a function' and 'confusion between univalence and injectivity'.

Part 2 dealt with a first attempt at testing the viability of the IFI. The IFI was implemented within a classroom setting with 27 participants. Participants then completed a short questionnaire to gather some qualitative data on their experience of the IFI. The analysis of this data looked for indications whether the IFI could have value by broadening the participants' thinking with respect to the function concept.

This article has the following structure. Following this introduction will be a section providing the theory of image functions, thus indicating how photographs or digital images can be considered as representing functions. That will be followed by a literature review which will firstly discuss the conceptual difficulties associated with the function concept in the context of secondary school and undergraduate level mathematics. Secondly, the literature review will discuss the APOS theoretical framework. It will end with the genetic decomposition of the function concept that was set up for this study. The literature review section is followed by a section that explains the details and workings of the IFI and then also proceeds to evaluate the soundness of the IFI from a theoretical perspective. This then concludes part 1 of addressing the research question. Part 2 follows and addresses the first implementation of the IFI and the qualitative analysis that was used as an initial viability check. Finally, the last section will bring together part 1 and part 2 and draw some conclusions.

\section{Theory of image functions}

Consider the photographs or digital images in Figure 1. In Figure 1a, the photograph of the horse consists of a finite number of pixels, or picture elements. This is easy to see in the zoomed image in Figure $1 \mathrm{~b}$ where we can distinguish individual elements of the eye of the horse. To each position in the image, a unique colour, Figure 1a, or shade of grey, Figure $1 b$, is assigned. Therefore, we can interpret these images as functions. 


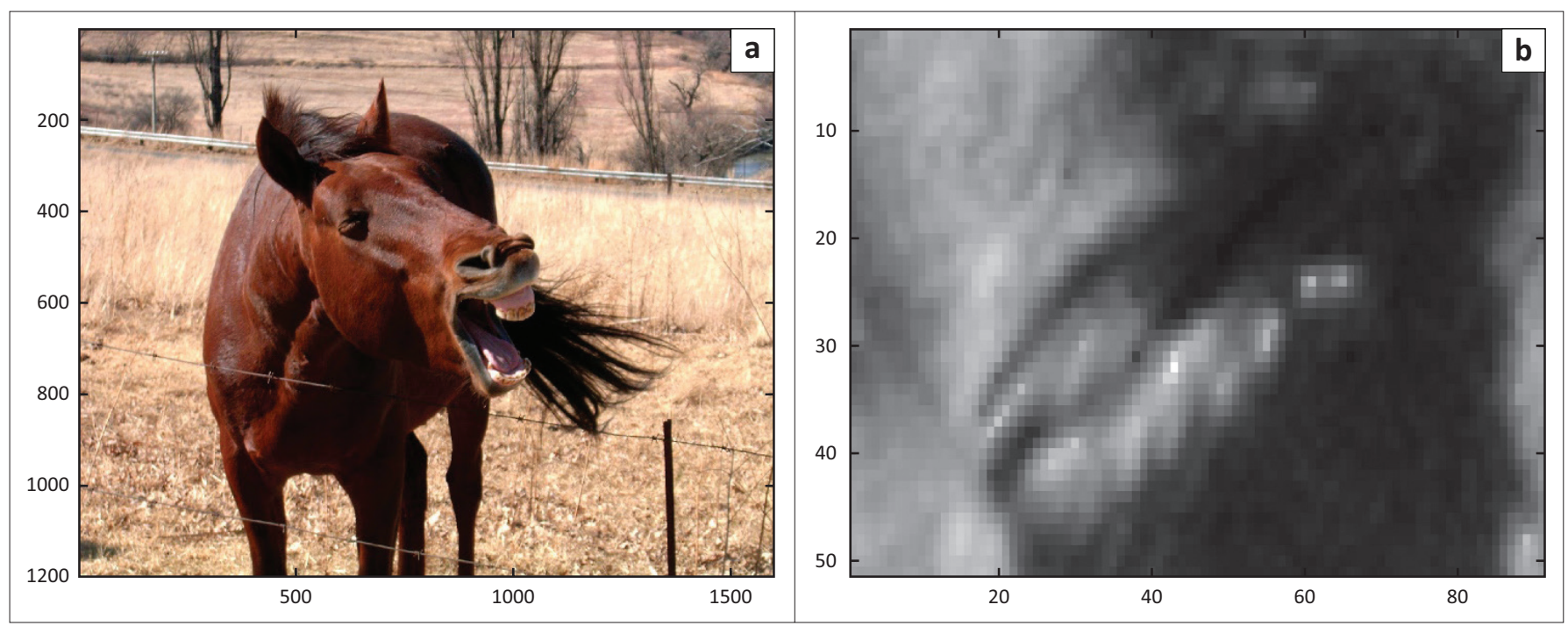

FIGURE 1: (a) Photograph of a horse. (b) Zooming in on the horse's eye, viewed as a greyscale image.

\section{Defining the image function}

An image function, $f(x, y)$, is a function with both $x$ and $y$ being positive integers (Gonzalez \& Woods, 2017). Any combination of such an $x$ and $y$ will form an ordered pair that will denote the position of a particular pixel in the image. Corresponding to each ordered pair is a unique colour. Typically, the different colours are represented using the RGB (red, green, blue) colour space. Any specific output of an image function is then an ordered triple providing the specific combination of red, green and blue. Typically, a scale of 256 different shades of red are used and the same for green and blue (Gonzalez \& Woods, 2017). If we then let the first shade be represented by 0 , the last shade would then be represented by 255 . Using these typical values, $256^{3}$ combinations of red green, and blue are possible. For example, the triple $(255,0,0)$ will be bright red as it contains the full complement of red and zero contributions of green and blue. $(255,255,0)$ is bright yellow, $(0,255,0)$ is bright green and $(57,229,212)$ would be called turquoise by some.

If we only consider the possible outputs where the three components of each triple are equal, we end up with what is commonly referred to as a greyscale image, where outputs are shades of grey. For example, $(0,0,0)$ is black, $(255,255,255)$ would be white and $(30,30,30)$ would be a dark grey. The image in Figure 1(b) is an example of a greyscale image. As the three values in each triple will be equal, the outputs for greyscale images each consist of a single number that represents the light intensity at a particular pixel.

An example: Consider Figure 1b, the greyscale image of the horse's eye.

Domain: This image has exactly 51 rows and 91 columns. The domain of this image function, is the set of ordered pairs: $\left\{(x, y) \mid 1 \leq x \leq 51,1 \leq y \leq 91, x \in Z^{+}, y \in Z^{+}\right\} \cdot Z^{+}$is the set of positive integers.
Range: The word range can refer to two different concepts, namely the codomain and the image of the function, so care should be taken in using it. The codomain for a greyscale image is easily specified as the set $\left\{s \in Z^{+} \mid 0 \leq s \leq 255\right\}$. This is then the set of shades of grey from which any greyscale image could be 'choosing'. When the term range is referring to the image of the function, it will consist of all shades of grey actually present in the particular 'picture'. Here then the image of the function and the picture-image of the function are the same set. The picture set would normally have repeated values or colours and would thus be a different multiset from the function image.

Consider more aspects of image functions.

\section{Surjectivity and injectivity of image functions}

An image function would seldom be surjective. With colour images using the RGB colour space, we have a total of $256^{3}=16777216$ unique elements in the codomain and most often many of these colours would not be present in the image. Being closer to surjective is normally desirable when it comes to images, as this would generally mean the image has higher contrast. Greyscale images typically have (only) 256 unique elements in the codomain; thus, being surjective has a much higher probability than in the case of colour images. It is clear that most images would not be injective either because it is highly probable that different pixels have exactly the same colour or shade of grey.

\section{Existence of the inverse function}

As for all functions, the inverse will exist if the function is injective. In the previous paragraph, we saw that it is highly improbable for an image function to be injective and consequently it is highly improbable for the inverse to exist. With the high resolution of modern cameras, it is quite common for digital images to consist of millions of pixels. For greyscale images of such high resolution, it would then be impossible to have an inverse, as greyscale images only 
have 256 output options available. Even for colour images with 16777216 possible output options, it will still happen often that at least two pixels will have the same colour. Therefore, the probability of the inverse existing is small.

\section{Continuity}

Consider any point $\left(x_{0}, y_{0}\right)$ in the domain of our image $f(x, y)$. Then we can show that:

$\lim _{(x, y) \rightarrow\left(x_{0}, y_{0}\right)} f(x, y)=f\left(x_{0}, y_{0}\right)$

Therefore, the image is continuous on its domain.

Proof: Let $\in>0$. Let $0<\delta<1$. If $\left|\sqrt{\left(x-x_{0}\right)^{2}+\left(y-y_{0}\right)^{2}}\right|<\delta<1$ then $f(x, y)=f\left(x_{0}, y_{0}\right)$ because the domain of $f$ is a subset of $Z \times Z$. Therefore, $\left|f(x, y)-f\left(x_{0}, y_{0}\right)\right|=0<\epsilon$. As the limit exists at any point in the domain and the limit is equal to the function value at that point, the function is continuous at any point in its domain.

\section{Differentiability}

A digital image is not differentiable at any point, yet a discrete derivative in the form of a difference quotient plays an important role in image processing. Applications where sudden changes such as steps, ramps, edges, lines or isolated dots need to be identified or accentuated often rely in part on some discrete implementation of a derivative. From calculus, we know that the derivative of a constant is 0 , which translates to the important requirement of derivative-based filters to give back a small or even zero response in a homogeneous region of an image. See for example Gonzalez and Woods (2017) for more on the implementation of derivative filters and, for example, Shrivakshan and Chandrasekar (2012) for more on edge detection techniques through the use of derivative filters.

\section{Literature review}

In this section, the focus will firstly be on the conceptual difficulties associated with the function concept as reported on in the literature. Secondly, the theoretical framework for this study will convey a background on the APOS theory and also portray the preliminary GD of the function concept. All these aspects are needed to guide the evaluation of the activities of the newly designed IFI.

\section{Conceptual difficulties of the function concept}

The concept or notion of a function is in its essence quite abstract but is often understood at a level where much of the abstract nature is not truly comprehended or might even be entirely lost. A student might for example directly equate the function concept to the existence of a formula (Dubinsky \& Wilson, 2013; Sierpinska, 1992; Vinner \&
Dreyfus, 1989). One of the prominent indications of a lack of depth in the understanding of the function concept is the restrictiveness applied to what constitutes a function. If a student starts to fixate on particular types or certain representations, they lose much of the richness of the function concept.

Being able to recognise a certain formula or graph as (representing) a function is of course a necessary skill, but not sufficient in providing the student with the correct concept aspects and cognitive reasoning to be able to grasp and utilise higher mathematical concepts. For example, something as immediate as the inverse of a function, concepts such as limits, derivatives and not forgetting ideas that are even more abstract, such as topological homeomorphism and category theory, remain out of reach. Thompson (1994, p. 39) argues that a fundamental difficulty is students' lack of connections between the various representations of the same function. What is it that is being represented? Thompson names this 'something' the 'core concept of function', that which is left unchanged when moving between the different representations.

What it boils down to is that students sit with an inadequate or erroneous function concept image. According to Tall and Vinner (1981, p. 151), the concept image constitutes the 'total cognitive structure that is associated with the concept'. This entails all definitions, properties, ideas, theorems and examples that a student has grouped under the heading of function over their mathematical career so to speak. Although a student may know the formal definition of a function, when exposed to a problem, the full concept image will be utilised to solve the problem. Doorman et al. (2012, p. 1245) also consider the concept image important and had as one of their specific goals the overcoming of a 'too-limited' function concept image. They investigated a new learning arrangement incorporating a computer tool to foster the transition from an operational to a structural view of functions. Within the particular setting, they report some success in overcoming difficulties with integrating operational and structural aspects and providing an explorative environment with respect to the aspects of covariation (Doorman et al., 2012, p. 1262).

As the function concept is fundamental, yet misunderstood, the suggestion is that students should be introduced to the idea in such a manner that the resulting concept image will be as rich and accurate as possible. It is in these respects that the exploration of digital images considered as functions, or image functions for short, could be particularly useful.

Much research has been done in confirming the difficulty with the understanding of the function concept and addressing this difficulty. Recent research includes that of Chimhande, Naidoo and Stols (2017), which confirms that the difficulty is prevalent at school level. They showed that the mental constructions were typically at the action level of understanding, which is the lowest level according to 
the APOS theory (Arnon et al., 2014). Doorman et al. (2012) explored the use of computer tools in aiding the transition to a structural view of function, that is, the object level of understanding. Makonye (2014) also provides a theoretical analysis focusing on the use of multiple representations to foster a nuanced concept image through approaches where the function concept is kept embedded in students' reality as far as possible. Other research focuses on specific aspects of the function concept and not on functions in general. Bansilal, Brijlall and Trigueros (2017) explored pre-service teachers' understanding of injections and surjections through an APOS study. They found that most participants were at the action level. Maharaj, Brijlall and Govender (2008) explored the use of instructional design worksheets in advancing pre-service mathematics students' understanding of the concept of continuity of single-valued functions. They found most participants were able to construct internal processes to make sense of continuity.

This article discusses a new pedagogy. This refers to the new approach reported on in this article, which used the field of digital image processing to gather 'mathematics for teaching' (Hoover, Mosvold, Ball \& Lai, 2016, p. 4). The specialised mathematical knowledge concerning image functions was used in a novel approach to the problematic teaching and learning of the function concept.

\section{Theoretical framework}

Working with constructivist ideas, essentially that learning is built upon previous learning, Dubinsky and McDonald (2001), as well as others before them such as Breidenbach et al. (1992), formulated the APOS framework (Arnon et al., 2014) for modelling the learning of mathematical concepts. Using the APOS framework, the development of the function concept can be modelled where the conceptualisation passes through stages in a non-linear way, generally starting with actions (A), then processes $(\mathrm{P})$, objects $(\mathrm{O})$ and finally mental schemas $(\mathrm{S})$. The non-linear here refers to the notion that the learning does not exclude the possibility of moving along different paths between the stages. For example, when busy conceptualising the function concept at the process stage, it might be useful or necessary to rethink and expand on one's conceptualisation at the action level.

At the action stage of understanding, an external stimulus such as an expression or an equation is needed to proceed, and the student cannot yet work with the concept entirely in the mind. In terms of image functions, we shall see in activity 1 of the IFI that explicit instructions are given to create a new image, one pixel at a time, by shading cells in a grid. At the process stage, the student can manage to construct the concept in the mind and also think about the underlying actions that make up the process, without actually performing any of these actions. In terms of image functions, the function as a process is realised when one can imagine how the possibly millions of pixels all get their respective colours (values) at the same moment when the photograph is taken. The number of pixels determines the domain, and which colours they potentially can get determines the codomain. When the student is at the object stage, the process has been encapsulated. The student is then able to think about other actions and processes that might be performed on this object. The student has then gone from an operational view to a structural view. In terms of image functions, the object level makes it possible to think about transformations, like contrast stretching, that can be applied to images. Images can be mentally grouped according to certain criteria and as such form sets of functions. At the schema stage, a student will be able to move freely between considering and using the concept as an action, process or object (Arnon et al., 2014, p. 30; Asiala et al., 1996, pp. 7-8). When considering which transformations would potentially enhance an image, the thinking is mainly at the object level, but when it comes to planning and performing the transformation, the thinking must be at the process and action levels.

The APOS theory can help us understand how the learning takes place by explaining what we see when participants are trying to 'construct their understanding of a mathematical concept' (Dubinsky \& McDonald, 2001, p. 1). The proposed mental structures needed for learning a mathematical concept are captured in what the APOS theory refers to as the GD. This GD is the theoretical blueprint against which the intervention of this article, the IFI, was measured.

\section{The genetic decomposition of the function concept}

Genes are the building blocks of life and so to determine a GD of a mathematical concept is to break down the learning of the concept into its imagined building blocks. The word imagined is used here as, in following the APOS theory, the breakdown is, among other things, dependent on the researcher's own knowledge (Dubinsky, 2000, p. 2; Maharaj, 2010 , p. 42). The researcher would use personal experience, completed research and observations to imagine and create a set of necessary mental structures and mechanisms at the action, process and object level. These structures and mechanisms are what someone who is learning the concept could need and use along the path of conceptual understanding (Arnon et al., 2014). Having the mental structures available makes it possible to judge at which level of conceptualisation a particular person is, with respect to a specific mathematical concept.

Keeping to the analogy of building, if the GD describes the progressive structures of the mathematical concept (the building), then the support needed to reach these structures would be described as the scaffolding. Part 1 of addressing the research question of this article deals with the evaluation of the intervention, that is, to ensure that the intervention is appropriate and sound. It must be 
appropriate and sound on two fronts: (1) addressing the mathematical content in line with the GD and (2) as scaffolding to support the student's 'construction of knowledge and skill' (Bakker, Smit \& Wegerif, 2015, p. 1048).

From Arnon et al. (2014, p. 27) we get the formal definition:

A genetic decomposition is a hypothetical model that describes the mental structures and mechanisms that a student might need to construct in order to learn a specific mathematical concept.

Necessarily we then need to define what a mental structure is. Again, from Arnon et al. (2014, p. 26):

A mental structure is any relatively stable structure (something constructed in one's mind) that an individual uses to make sense of mathematical situations.

The GD given in Table 1 is based on the decomposition given in Arnon et al. (2014, p. 29). Extensions and expansions are based on the researcher's own experience complemented by current literature on the topic.

The GD given in Table 1 conveys the mental structures of the function concept at the action, process, object and schema levels. Furthermore, it also describes the mechanisms of progression, namely Interiorisation, Encapsulation and Activity. In the APOS theory, these mechanisms are the proposed means by which one can transition from one level to the next level of conceptualisation (Arnon et al., 2014, p. 16). Based on a literature review, Table 2 provides further criteria that can indicate when and when not a student's understanding of the function concept can be considered at the action, process or object stage of conceptualisation. Alongside the GD of Table 1, these indicators were also used in the theoretical evaluation of the soundness of the IFI. In Table 2, reference is made to the activities of the IFI that are linked to the particular conceptual indicators given in brackets, for example A2 or P3. Details can be found as part

TABLE 1: Preliminary genetic decomposition of the function concept.

\section{Concept}

Action

From Action to Process: Interiorisation

Process

From Process to Object: Encapsulation

Object

From Object to Schema:

Activity

Schema

\section{Description}

Take an element of one set and apply an explicit rule, typically an (algebraic) expression, to determine a unique value belonging to another set.

Repeating this action, especially with sets with different kinds of elements, starts the interiorisation by helping the student to reflect on the action and to see the pattern of choosing from one set, the domain, then doing something and then obtaining something else. Special emphasis must be placed on getting the student to consciously think about the chosen and the determined 'somethings' as belonging to specific sets. This is necessary, placed on getting the student to consciously think about the chosen and the determined 'somethings' as belonging to specific sets. This is necessar situation. This also fits with the action level as someone at this level needs external stimuli (Arnon et al., 2014, p. 30; Asiala et al., 1996, pp. 7-8) to conceive of a function and consequently also needs this external stimuli to start reflecting on the concept.

A dynamic transformation of inputs in the domain to outputs in the range without any explicit calculations needed.

When conceiving of the function as a process, it can become necessary to think about applying an action or even a different process to this function. The function as process needs to be made static. The process (the function) needs to be captured and seen in its totality. Doing this encapsulates the function to become the function conceived of as an object (Arnon et al., 2014, p. 30; Asiala et al., 1996, p. 8).

Identify the word function as a noun. A noun has properties that can be listed. The noun is described by adjectives. A function could be, for example, rapidly changing, smooth, constant, etc. Furthermore, being able to think of the function process in its totality and imagine and construct actions and processes that can transform this totality (Bansilal et al., 2017, p. 24).

'A schema is only constructed when it is functioning and it only functions through experience: then that which is essential is not the schema as structure in itself but the structuring activity that gives rise to schemas' (Piaget 1975/1985, as quoted in Arnon et al., 2014, p. 110).

A dynamic mental framework, which a person might not be consciously aware of, that describes the function concept as simultaneously existing as an action, a process and an object and that links and relates these different underlying mental structures. A person evokes their schema when confronted with a problem involving the topic of functions. Specific examples of functions such as rational or trigonometric functions along with their properties and relations will also be included in the schema.

TABLE 2: Indicators and counter-indicators of APOS level attainment with respect to the function concept.

\section{General definition}

\section{Action}

A student at the Action level is restricted to transformin mathematical objects by using external prompts such as formulas or expressions. Steps or instructions are needed (Asiala et al., 1996; Dubinsky \& Wilson, 2013).

IFI activities:

Activity 1 - Follow explicit instructions to shade cells in a

grid on paper and answer reflective questions. (A1, A3)

Process

A student at the Process level constructs the function mentally and can realise the complete transformation of elements from the domain to elements of the range.

No external prompts are needed (Arnon et al., 2014

Dubinsky \& Wilson, 2013)

\section{IFI activities:}

Activity 2 - Experiment with different output values for the image function using the software Paint. (P2)

the image function using the software Paint. (P2)

and also consider the uniqueness property, being

and also consider the uniqueness property, being

injective, being surjective and the existence of an inverse. (P1, P2, P3, P4)

\section{Object}

Through transforming the process by actions and other processes, the process is encapsulated to become an object. The dynamic process becomes a static entity (Arnon et al., 2014; Asiala et al., 1996; Dubinsky \& Wilson, 2013).

IFI activities:

Activity 4 - Explore function composition in order to enhance the contrast of images. $(01,02)$

\section{Conceptual indicators of the leve}

A1 Able to substitute numbers into an expression and calculate (Breidenbach et al., 1992).

A2 Able to compose functions given by simple formulas (Breidenbach et al., 1992).

A3 Can recall the definition of a function (Breidenbach et al., 1992).

P1 Understands general composition of functions and reversal of functions (Dubinsky \& Wilson, 2013).

P2 Can provide a definition of a function that includes mention of the inputs, outputs and a rule (Arnon et al., 2014).

P3 Can realise which steps of the process can be swapped or even left out for particular cases.

P4 Able to determine whether a function has an inverse as the reversal of a process (Arnon et al., 2014).

01 Able to think about and convey the global properties of a particular function or a type of function, for example periodic, smooth, or constant, monotonic.

O2 Able to form sets of functions, perform operations on functions and even 'construct a function that is a limit of a sequence of functions' (Arnon et al., 2014).

\section{Counter-indicators of the level}

Before the action stage, a student is said to exhibit a pre-function response to questions such as: 'What is a function?' (Breidenbach et al., 1992). No useful conveying of function ideas is present.

- Difficulty interpreting a situation as a function unless a formula is given (Asiala et al., 1996)

- Function composition is too difficult in atypical situations, for example in the absence of formulas or with piece-wise defined functions (Breidenbach et al., 1992).

Commonly not using the definition when confronted with problems (Tall \& Vinner, 1981).
Cannot easily move between different representations of the function.

- Considers a piece-wise defined function as consisting of multiple functions.

- Struggles to create a function example that is connected with some real-life situation (Chimhande et al., 2017, p. 5). 
of the individual discussion of each IFI activity in the next section. Table 2 provides indicators only up to the object level to align with the content and intentions of the IFI.

As was discussed in the introduction, what follows now is a two-part approach. In the first part, the IFI will be introduced and evaluated to see if it is theoretically sound. The second part will report on the first implementation of the IFI and the subsequent gathering and analysis of qualitative data with the purpose of looking for any indications that the IFI might be viable with respect to the learning of the function concept.

\section{Part 1: The Image Functions Intervention and its theoretical evaluation}

The IFI was created using e-learning authoring software which delivered it as a package that could be uploaded into the learning management system (LMS) used by the local higher education institution. This allows a student to complete the IFI on a computer at their own pace without any lecturer involvement.

As a general context, the IFI deals with finding a missing student of which one recent photograph was available on the student's Facebook page. This photograph, however, was taken in low light conditions and as a result needs some processing before it will be helpful in finding the missing student. This theme runs like a story throughout the intervention. This theme was chosen as students are familiar with the context, they can easily understand the contingency relationships involving the variables that are present and they are generally interested in the type of context (Donovan \& Bradsford, 2005, p. 359; Eggleton, 1992). Besides this storyline, the IFI conveys the theory of image functions as was discussed in the previous section. The theory is interwoven with reflective questions and specific activities. These activities are meant to keep in line with the following three principles:

- Activities directly link with the mental structures determined in the GD of the function concept given in Table 1 and the conceptualisation indicators in Table 2 (Salgado \& Trigueros, 2015, p. 107).

- Activities address the categories of conceptual understanding. Dubinsky and Wilson (2013, pp. 85-86) grouped the most common conceptual difficulties associated with the function concept, as they had found in literature, into categories.

- Activities form an experiential base for the aspects of the function concept to be studied (Dubinsky \& Wilson, 2013, p. 90).

The principles given above will be used as the criteria for the theoretical evaluation of the IFI. Each of the IFI's four activities will be discussed and evaluated with respect to the first of the three design principles given above. The subsection following this one will evaluate the IFI with respect to the other two principles.

\section{Activity 1}

Description: The student is asked to draw an $8 \times 8$ grid on paper as in Figure 2a. The student must then take a pencil and shade the blocks at positions B2, B3, C1, D1, D5, D6, D8, E1, E4, F2 and F3. Accurate shading leads to the result given in Figure $2 b$.

Students are asked to reflect on the activity by letting them provide answers to questions pertaining to uniqueness aspects of functions and asking questions to let them think about the choices that can be made with respect to input and output.

Questions asked as a part of this activity:

- Now that you have created your image, would you say that the colour at any specific position in your image is unique?

- If you choose a position in the image, what will you find there?

- Are you free to choose any position in the image?

- Once you've chosen a position in the image, can you then also choose which colour will appear there?

- So is it true that the colour is dependent on the position?

To keep the activity and the IFI interactive, students are required by the software to first answer the questions before being able to proceed. The question formats vary between multiple choice and typing an answer. From this activity, there is a natural flow in letting the student discover that a photograph or digital image can be interpreted as a mathematical function.

Connecting to the GD and the conceptualisation indicators (CIs): This activity aims to connect the repeated action of assigning a shade of grey to a specific position in the grid to a function value that is assigned to specific input. This activity is therefore aimed at helping students to construct the function concept as an action, which is the first stage of understanding according to the APOS theory. By repeating the shading action, an attempt is made at facilitating the interiorisation mechanism as was described in the GD. The instructions of this activity connect with the external prompts (see the CIs) or explicit steps needed by a student whose understanding is at the action level. The reflective questions of this activity

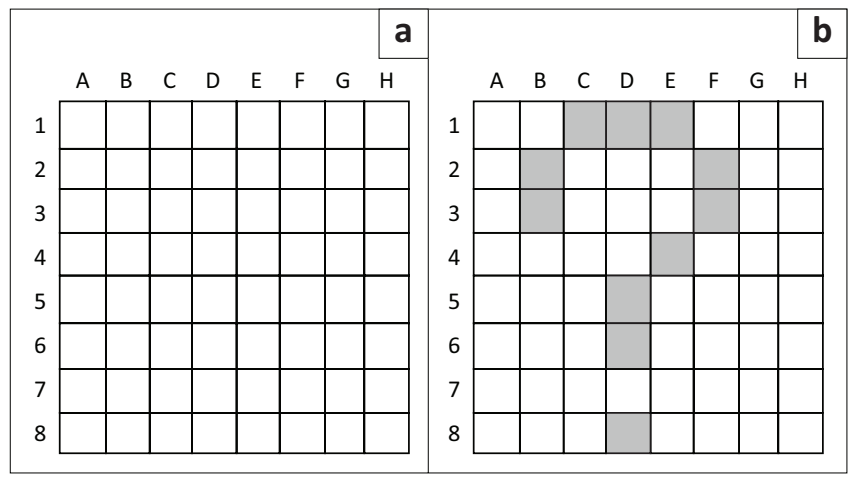

FIGURE 2: (a) An empty $8 \times 8$ grid. (b) Result obtained at the successful completion of Activity 1. 
connect with the CI criteria that the student will know the definition of a function and will as such be led to discover that an image can be interpreted as representing a function.

\section{Activity 2}

Description: Students visualise some function outputs by using the colour editor in the commonly available software Paint. Students are prompted to try different combinations of red, green and blue and they can then see what those combinations look like. This is an easy-to-use experiential playground where students are asked to try out for example negative values or non-integer values or values larger than the maximum used in the colour scheme and then observe what the software does to these inputs. The software automatically adjusts an inappropriate input in a default manner. If a negative value is typed, the value is changed to the absolute value. If a number larger than 255 is typed, it is automatically changed to 255 . If a non-integer is typed, it is changed to the last digit that was typed.

Connecting to the GD and the CIs: This activity allows students to engage with the idea of the range of an image function practically. At a later stage, students must also think about the domain by realising that the image only has a finite number of rows and columns. The concept of the domain of a function is thus a quite practical 'thing' with respect to image functions. This activity connects to the GD mechanism of interiorisation to assist in going from an action understanding to a process understanding. Furthermore, it connects to the CIs by attempting to broaden the student's understanding of the definition of a function, by emphasising the necessity of the domain and range. This is necessary for a process understanding of the function concept.

\section{Activity 3}

Description: Students are brought back to the image function formed in Activity 1, but now both the rows and columns are depicted by positive integers as in Figure 3.

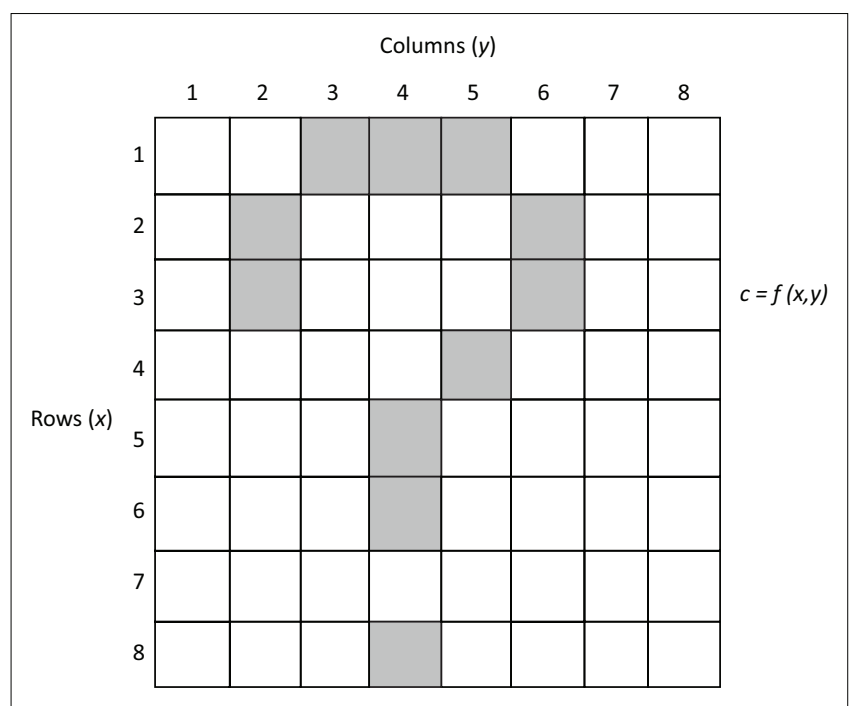

FIGURE 3: The function regarded as a process.
Reflective questions are used in this activity to assist students in constructing the function concept as a process:

- Does the choice of the first block you coloured or the sequence in which you coloured the blocks make any difference to the final image?

- What is the difference between how you formed this image and how an image would be formed by your cell phone's camera?

- Does your camera also take one input at a time and 'fill' in the colour for that position?

After a student completes these questions, a description is given comparing the point-by-point, successive way in which they shaded each individual block to what will happen inside a camera. Inside the camera all the 'blocks' get shaded simultaneously. The function is then a process of taking the entire domain at once and filling it with the range. Students are again led to think about the specifics of the domain and the range:

- Thinking about the inputs

- Does $f(2,3)$ make sense?

- Does $f(2,3.561)$ make sense?

- What conclusions can be drawn about the set of allowable inputs?

- What do we call the set of inputs for a function?

- Thinking about the outputs:

- In a grayscale image can the value at any position possibly be described as 'blue'?

- In a colour image, can the value at any position possibly be described as 'dark grey'?

They are also led to think about the uniqueness property and the aspects of being injective and surjective:

- For the image function in Figure 4, the codomain is \{red, purple, green, blue, black, pink, orange, white, yellow\}.

- What is the range of this image function?

- Is this image onto?

- Is this image one-to-one?

Finally, thinking about the inverse process is also introduced here, for example questions such as:

- Yellow is a colour that is present in this image (Figure 4). If you now make yellow the input, what will be the output?

- Do you think the image function shown will have an inverse?

Connecting to the GD and the CIs: As described in the CIs, at the process level a student should be able to mentally construct the function as the complete transformation of the domain to the range. This idea is captured in how the camera captures all of the pixels' values simultaneously. This activity also explicitly deals with the aspects of the reversal of the function process and the existence of an inverse and confronts students with a fuller grasp of the definition of a function while at the same time having no formula. Having a function without a formula was also addressed in Activity 1, but as it is one of the common 


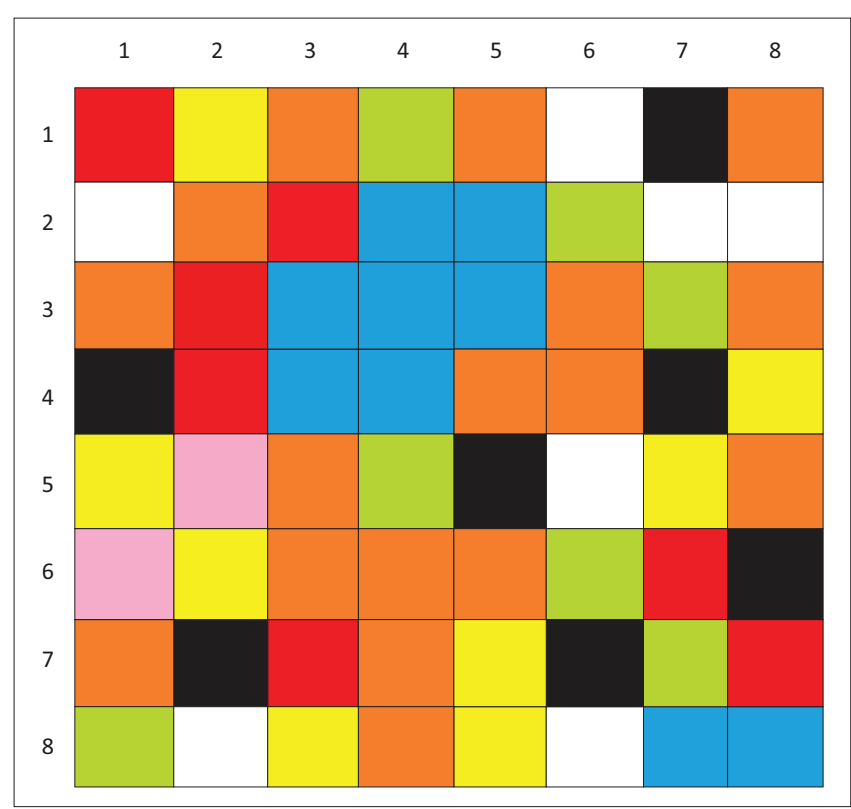

FIGURE 4: Will this function be injective?

conceptual difficulties associated with the function concept (Dubinsky \& Wilson, 2013, p. 85), it is valuable to address it again.

\section{Activity 4}

Description: With this activity, the aim is to assist students in constructing the function concept as an object. The story in which the intervention is set is brought to a peak here. The student sees how the knowledge of image functions, together with simple contrast stretching, is used to enhance the photograph discussed at the start of the intervention. It is enhanced to such a degree that sufficient information can be gathered from the image to assist in identifying the place where the photograph was taken. The contrast stretching seen in this activity is achieved through function composition.

As an introduction, the activity lets the student explore contrast stretching with pen and paper. The student is asked to draw the $5 \times 4$ grid as pictured in Figure 5a.

The number of vertical lines, $v$, in each cell (pixel) can be considered the colour of that cell. Figure $5 \mathrm{a}$ is then an image function, say $f(x, y)$. The student is then asked to draw a second $5 \times 4$ empty grid and then fill in its values by applying the function $g(v)=4 v-4$ on the original image of Figure 5a. A new image function $h(x, y)$ is thus created through function composition. We obtain Figure $5 \mathrm{~b}$ through the function composition $h(x, y)=g(f(x, y))$. For example, $h(1,2)=g(f(1,2))=g(2)=4(2)-4=4$. This composition stretches the contrast to such an extent that we can now see the number 5 or the letter $S$ present in the image. The 5 (or S) was of course already present in the original image, but it was difficult to distinguish it from its background. It was difficult to distinguish due to the low contrast of the original image.

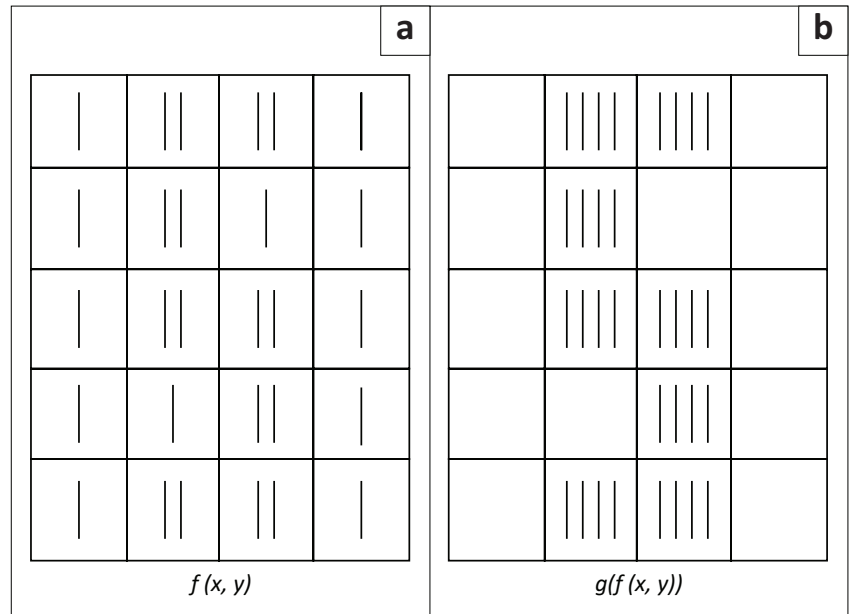

FIGURE 5: (a) Low contrast image. (b) Increased contrast after function composition.

Once the introductory contrast stretching is completed, the student is brought back to the photograph associated with the missing person's case. This photograph is seen here in Figure 6a.

Students are initially asked to describe in their own words what is wrong with the photograph, or consequently the (image) function in Figure 6a. How can a function be 'wrong', or for that matter be described? As an object, the function acquires global properties such as having low contrast, therefore a small average difference between adjacent pixels across its domain.

This activity lets a student realise that a low-contrast image can be improved by regarding it as one single thing - an object - that can be transformed by another function. Function composition is used to transform the original image function, $f(x, y)$, into a new and improved image function $g(f(x, y))$.

$$
n e w_{\text {object }}=g\left(\text { old }_{\text {object }}\right)
$$

Connecting to the GD and the CIs: If we carefully design the transformation function, $g$, we can obtain the desired results and again describe the new function as a whole. A function then becomes a noun and a noun can be described by adjectives. We might say that the new function is beautiful, it is clear, it has high dynamic range, it is smooth or, as was planned for the image of the intervention, it has improved contrast as can be seen in Figure 6b. This ability of seeing the function as a whole and not as something that you do, but rather something that can be acted on, is described in the GD at the object level. The function composition used in this activity fulfils the role of a process acting on the function process. This is described as encapsulation in the GD and is also a requirement in the CIs. Through acting on the function as object and reflecting on properties of the function such as contrast or brightness, a student could start to realise that image functions are a type of function, similar to how exponential or trigonometric 


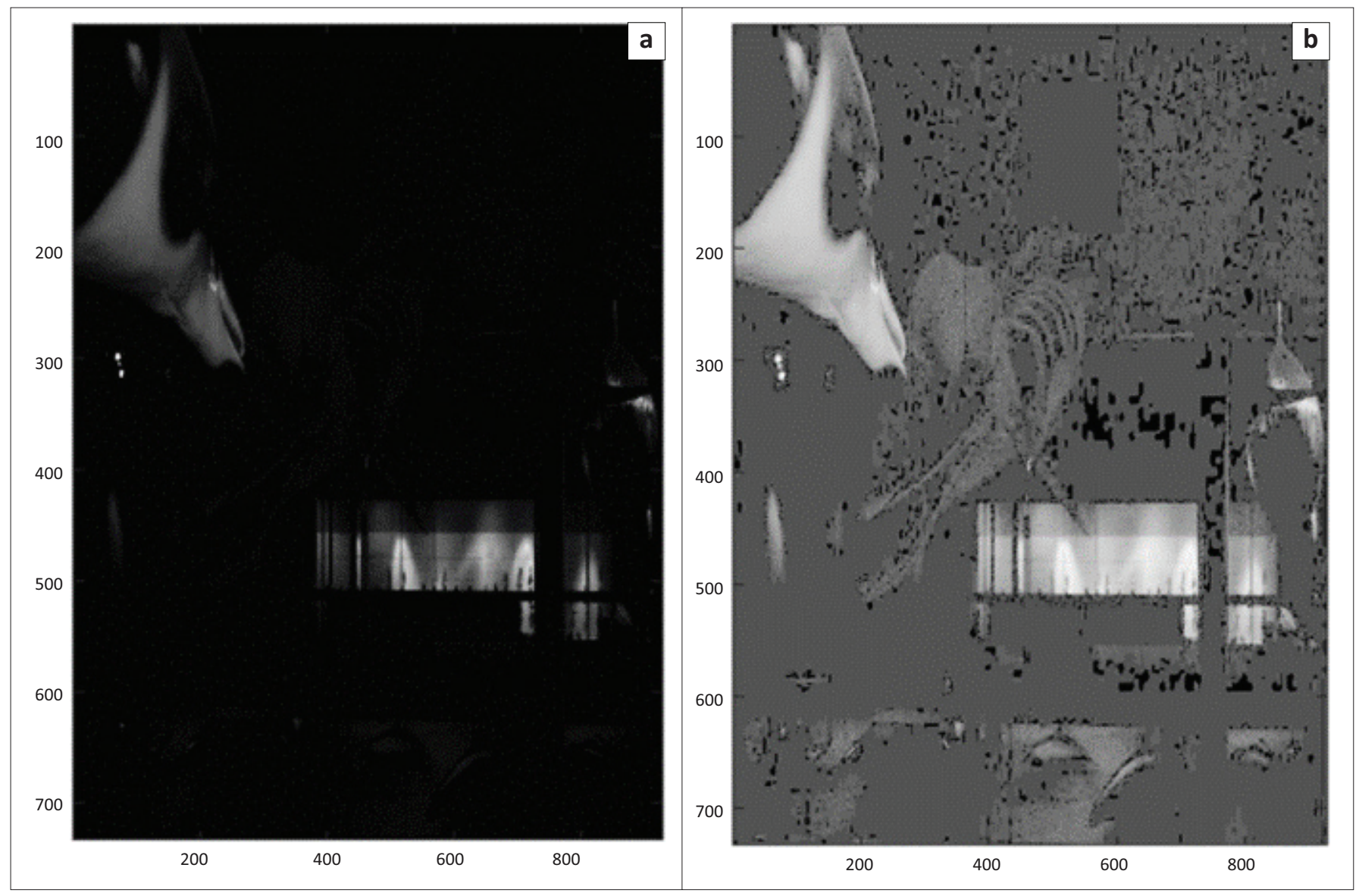

FIGURE 6: (a) Low contrast image. (b) Increased contrast after function composition

or linear functions are different types of functions. This connects to the $\mathrm{CI}$ of being able to think about and convey the global properties of a type of function. Furthermore, a student can be led to grasp that different photographs of for example the same scene or person form a set of image functions. Mentally constructing a set with functions as its elements is a further indicator from Table 2 of being at the object level of conceptualisation with respect to the function concept.

\section{Expanded theoretical evaluation of the Image Functions Intervention with respect to the first principle}

The IFI follows the APOS general trajectory in the sense that information and in particular the activities are ordered to first let students retouch on the function concept as an action, then move on to a process and then finally to the function as an object. However, this trajectory is not the only possible path through the levels. As was discussed in the theoretical framework, in general the learning can pass through the APOS stages in a non-linear way (Arnon et al., 2014). In the discussion of the four activities before, it was already indicated how the activities aim to guide the student to construct the desired mental constructs that emerged in the GD and link to the CIs indicators of Table 2. To expand on the previous evaluation, we now track the GD while taking into account the IFI's underlying path through the APOS stages: action, process and finally object.

\section{Action}

At the action level, a student is expected to take an element from the domain and find its corresponding value from the range. This is what is required in Activity 1. Repeating the actions (Asiala et al., 1996:7; Dubinksy \& McDonald, 2001:3) together with encouraged reflection about the actions and the involved sets (expanded on in Activity 2) triggers the necessary interiorisation mechanism to lead the student to a process understanding of function.

\section{Process}

At the process level, a student must now be able to capture the creation of the image of the function mentally (in the sense of the range) as a whole. Possibly infinitely many function evaluations can be imagined taking place simultaneously in the mind. Dubinsky and McDonald (2001, p. 3) describe that at the process conception, the individual can think about infinitely repeating the same kind of action, as no external stimuli - such as following the steps of a formula - are still needed. Activity 3 assists here by juxtaposing the point-by-point creation of a photograph by individual actions with the actual chemical or electric process that is going on inside a camera to form the photograph all at once. Inside the camera, the entire film or sensor is illuminated and so all pixels get their values at the same time. 
The action of one 'element of light' reaching one point on the film or sensor to make one specific colour at that point or pixel can be imagined to be repeated simultaneously for all the millions of pixels in the eventual photograph.

\section{Object}

At the object level, a student must now be able to grasp a function as one static entity. Activity 4 aids the encapsulation of the former dynamic transformation process to a 'thing', to become a noun with associated adjectives and properties. The encapsulation of functions at the process level by performing actions or other processes on these functions is reported to be key to transitioning to the object level of understanding (Asiala et al., 1996). Yet, this type of encapsulation is missing from experience when it comes to functions (Asiala et al., 1996, p. 8). In the IFI, this is exactly the type of encapsulation emphasised by means of the function composition in Activity 4. Actions and processes are applied to the image function in order to achieve the specified goals.

As the intention with the intervention was only to look to improve understanding up until the object level, the schema level is not discussed in this analysis of the intervention.

\section{Evaluating the Image Functions Intervention with respect to the second and third principles}

In keeping to the second and third design principles, the IFI has to form a basin wherein the conceptual difficulties and other required concept aspects associated with the function concept can be explored in a familiar context. In the IFI, that context is created using photographs. To see if the IFI is compliant, we will look in turn at the most commonly occurring function concept difficulties (Dubinsky \& Wilson, 2013, pp. 85-86) and other function aspects:

\section{What constitutes a function?}

Using image functions encourages the realisation that a formula is not necessary to have a function. Vinner and Dreyfus (1989) categorised students' definitions of a function into six categories: Correspondence, Dependence Relation, Rule, Operation, Formula and Representation. Ultimately all six of these categories can be valuable viewpoints when dealing with various functions, function properties and applications. However, if a student focuses too much on the idea of a formula or an equation for a function, the construct could easily be cemented in their thinking that only formulas, or rules or correspondences having known formulas or equations, can be considered as functions. This kind of restrictiveness is quite common (Breidenbach et al., 1992; Dubinsky \& Wilson, 2013; Sierpinska, 1992; Vinner \& Dreyfus, 1989). From the start of the intervention, the student is put in a state of disequilibrium by introducing an everyday thing, such as a photograph, as a function. This state of disequilibrium, in the style of Piaget (Wadsworth, 1978, p. 80), is necessary here as the student's concept image of functions has been formed and re-enforced over a number of years already. Therefore, by throwing the student off balance, room is created for the restructuring of the concept image. It is the suggestion of this article that the IFI's reintroduction to functions via image functions can challenge the fixation and restricted thinking in terms of formulas and equations. This is necessary to develop beyond the action level.

Students also often focus on the symbols representing the variables, instead of the quantities they are representing. In the IFI, working with the image functions is done without the need for symbols. Familiar or intuitively understood terminology such as row, column and grey level are used.

Vinner and Dreyfus (1989, p. 361) also showed that any seemingly irregular behaviour such as discontinuity, a split domain or ' $[t]$ he idea that the graph of a function has to have a stable character' are erroneous ideas that students often use to disqualify some rules or graphs as functions. The IFI, by working with image functions, again has the advantage over using linear functions, for example in that images change character easily across the two-dimensional domain. This is even more apparent when the object conception of function has been reached after Activity 4. Therefore, students will not be left with the idea that a function needs to act 'nicely' in any way to be considered a function. In contrast, Carlson and Oehrtmann (2005, p. 2) mention the case of students thinking that constant functions are not functions 'because they do not vary'. Also see Bakar and Tall (1991). Confrey and Smith (1991) refer to the constant function as an example of a 'monster' function and confirm that students exclude it as a function because they expect a function to 'covary'. In working with the IFI's activities, it occurs naturally that portions of the image will have the colour or grey level stay constant over smaller or even large areas. A student can thus discover intuitively that an image function and, by extension, all other functions are allowed to display seemingly nonregular behaviour over certain portions of their domains.

\section{Univalence and injectivity}

In working with the image functions of the IFI, the oftenproblematic univalence property is made practical and simultaneously important. In addition, the confusion that is often seen (Harel \& Dubinsky, 1992) between the univalence property and the function being injective is addressed in a tangible way. In terms of image functions, it is evident that at any specific input (pixel) the photo has only one output (colour) and as such exhibits the univalence property. Furthermore, if we determine that for the particular photo, the colour at any pixel does not occur at any other pixel, we have determined that the photo or image function is one-to-one, thus injective. Reflections to evoke these realisations with students are delivered through Activity 3 of the IFI.

\section{Multiple representations}

The activities of the IFI provide opportunities to ask new questions about multiple representations of functions. The multiple representations idea garners plenty of attention in 
textbooks as well as research (Carlson \& Oehrtman, 2005; Confrey \& Smith, 1991; Stewart, 2015, p. 10). However, according to Thompson (1994, p. 39), students still miss that which stays unchanged between these representations: the 'core concept'. How would one represent an image function differently than by means of the photograph? Setting up a table that states explicitly the colour that belongs at each position can illuminate the fact that the representation itself is not the function. Students can be led to realise that similar to an equation or a graph, the purpose of a table or the photograph is to tell us the output that belongs to any specific input. Why do we then have or use multiple representations? We only use them if they help us to understand or analyse the function. A blind person could get no value from a graph of a function, but could there maybe be a way to listen to a function? Could an audio representation be created, and would it be helpful?

In validating the design from a theoretical viewpoint, we are moving the intervention towards a correct organisation of the knowledge to optimise deep understanding. This is key in developing sufficient expertise to solve problems that flow directly from the topics involved as well as related problems (Donovan \& Bransford, 2005, p. 16). Naturally, the theoretical validation will need to be followed by empirical study to provide practical validation. This is the content of research currently in progress.

For this article, qualitative data were gathered by means of a questionnaire, but not to validate the GD as is typical in the APOS theory (Arnon et al., 2014; Dubinsky, 1991). Rather, the purpose was to investigate the viability of the IFI. The next section reports on the first implementation of the IFI and the consequent use of a questionnaire and the qualitative data analysis.

\section{Part 2: A first attempt at determining the Image Functions Intervention's viability}

This section deals with the first practical implementation of the IFI. It forms part 2 of addressing the research question as was given at the end of the introduction of this article. Part 2 investigated the viability of the IFI by looking for indications that the IFI could have value through broadening the participants' thinking with respect to the function concept. Achieving this objective would give us proof of principle, that is, determining if the IFI is sensible and worthwhile to investigate further. Proof of principle, together with qualitative analyses indicating what the content of an intervention should entail, assists in determining the need and validation for further testing (Pressley, Graham, \& Harris, 2006, p. 7). A questionnaire was used to gather qualitative data on the participants' experience of the IFI. A qualitative method was used here to allow the exploration of participants' perceptions and allow for unexpected feedback on the IFI.

\section{Sampling and data collection}

The IFI was implemented in a classroom setting with a group of 27 students in a first-year Calculus course. This is not the intended method by which the intervention will be implemented, as it was designed to be a self-directed mini module where a participant follows their own pace and can actively engage with the various activities of the intervention. However, to gauge the initial reaction of participants to the material and activities, it was decided that a classroom setting, together with a questionnaire at the end, would be sufficient and still enable us to achieve the objective of a proof of principle.

This group was chosen for convenience but fulfilled the minimum criteria of having prior knowledge of the function concept. From casual observation, the group had male and female members and these members were from at least three different ethnic backgrounds.

The questionnaire was handed out to all 27 participants and it was made clear that participation was voluntary and would be anonymous. No personally identifying information was asked, as this was not deemed necessary for a proof of principle endeavour, thus simply testing if the IFI could be viable. The questionnaire consisted of three grammatically closed, but conceptually open, questions (Worley, 2015, p. 19) as this still allowed any elaboration the participant might wish to provide.

\section{Data analysis}

The method of content analysis with emergent coding was used (Maree, 2016, p. 111). The responses to the three questions were searched for any indications relating to the objective of the questionnaire. After reading the responses a few times, four themes were identified:

\section{What constitutes a function?}

Students commonly struggle with misconceptions with regard to what can and what cannot be regarded as a function (Dubinsky \& Wilson, 2013, pp. 85-86). More discussion on this topic was given as part of the theoretical evaluation of the IFI in Part 1.

\section{Functions are connected to real life}

From reading through the participant responses, it seems that many were almost surprised to find functions being used in such an everyday type of topic as photographs. This theme does not represent an improved understanding of the function concept but can possibly assist in making the topic interesting to participants. This interest can increase their motivation, which is key to effective learning (Eggleton, 1992, p. 1). Connecting the function concept knowledge to everyday experience also assists later retrieval and application (Donovan \& Bradsford, 2005, p. 364). 


\section{Domain and range}

This theme relates to the understanding that a function requires a set of allowed inputs and, associated to each input, a unique output. The outputs form a set as well. From the GD, we saw that clear understanding of domain and range are required to construct the function concept at the action level and to interiorise the actions to start constructing at the process level.

\section{Function inverses}

At the process level, being able to reverse the function and form the inverse function is required (Breidenbach et al., 1992).

Each response given to the three questions was read and reread in order to judge if it contained any indication that the participant experienced a broadened understanding of the function concept. The indication was then classified as belonging to one of the four themes.

\section{Validity}

It was not the intention of the questionnaire to deliver a generalisable result. The questionnaire was part of the effort to establish a proof of principle. Proof of principle can be interpreted as looking for proof that the IFI can have value, at least in some settings with some participants. To increase the credibility of the qualitative data analysis, colleagues were asked to independently verify themes, occurrences and results.

\section{Results of the qualitative data analysis}

In the data analysis section, four themes were discussed that emerged from repeated reading of the participants' responses. The responses were then analysed individually within the structure provided by the four themes.

This was done while keeping in mind that we were looking for indications that the participant experienced a broadened understanding of the function concept. In the analysis to follow, these three questions $(\mathrm{Q})$ were asked:

- Q1: Having completed the IFI, have you realised or learnt something in particular of the function concept?

- Q2: Is there an aspect of the function concept that is now clearer to you?

- Q3: Is there some aspect of the function concept that you might have thought about in some way before, but now realise that you were wrong about in some sense?

\section{Theme 1: What constitutes a function?}

The analysis illustrates that participants are showing an expansion of their 'concept image' (Tall \& Vinner, 1981, p. 151) related to what can also be considered a function. Consider the following responses:

'Yes, I realised functions have a broader meaning and that it forms a big part of our technological lives.' (Participant 1, answering Q1,)
'Yes, that all images are also functions.' (Participant 13, answering Q1,)

'Yes a function can be determined in different ways.' (Participant 21, answering Q1,)

'Yes, that not all functions are graphed on cartesian planes.' (Participant 18, answering Q3,)

\section{Theme 2: Functions are connected to real life}

Again we see participants' concept images expanded. Here it relates to a realisation that functions can be useful and specifically useful outside of mathematics itself. Some participants realise that functions can be part of their lived experiences. Consider the following representative responses:

'Yes, it can be used for various purposes.' (Participant 2, answering Q1)

'Functions can be used for a lot of purposes out side of maths [sic].' (Participant 5, answering Q1)

'Yes, I understand that math is used everywhere.' (Participant 6, answering Q1)

'Yes, how unclear images been processed to have clear pictures about something and I did not know that how functions are used in life.' (Participant 22, answering Q1)

'Yes. I didn't know functions can relate to real life experiences and applies to images and is involved in biometric scanners.' (Participant 19, answering Q3)

'Yes, I have learned that functions can be used for more than calculating or predicting change.' (Participant 25, answering Q3)

\section{Theme 3: Domain and range}

A few participants gave some indication of increased understanding of the aspects of input and output. Clarity on input and output, as relating to domain and range, would assist in transitioning from an action level to a process level of function conceptualisation. Consider the following responses:

'Yes there is a input and a output.' (Participant 4, answering Q1)

'Yes, there is an input and output.' (Participant 9, answering Q1)

'Inputs and outputs of a function.' (Participant 3, answering Q2)

\section{Theme 4: Function inverses}

Some participants reported to have gained increased clarity on the aspect of function inverses. To understand the function concept at the process level, one needs to be able to reverse the actions of the function and then progress to formulating the inverse function or deciding if the inverse function will exist. The following responses are representative:
'Yes, the differentiation between inverse functions.' (Participant 13, answering Q2)
'The derivatives or inverse of functions.' (Participant 15, answering Q2)
'Yes, inverse of a function.' (Participant 3, answering Q3)

\section{Discussion and conclusions}

In this article, our objective was to determine if the IFI could be considered a sound and viable tool with respect to the 
learning of the function concept. This objective was addressed in two parts.

The first part was a theoretical evaluation of the IFI. This evaluation strongly depended on a GD of the function concept. Not only can a GD serve as the basis for designing a learning intervention, it also serves to make the consequent analysis more reliable (Arnon et al., 2014, p. 38). Through empirical study, the success of the APOS theory has been shown through using the GD not only to describe the mental constructions of participants but also to design 'effective instruction' (Weller et al., 2003). The required GD was determined, showing the necessary mental structures at the action, process, object and schema levels. Connecting with the GD, indicators were set up (see Table 2) which could be used to judge the conceptual levels of a participant. Consequently, the activities of the IFI were shown to keep true to the GD and the indicators of Table 2. This was done by describing each activity and explicitly making connections between aspects of each activity and the requirements in the GD and the indicators of Table 2. Important to note is that the IFI provides opportunities for encapsulation of processes through the function composition used in its contrast stretching activities. In the APOS theory, encapsulation is the mechanism by which conceptualisation evolves from the process to the object level (Arnon et al., 2014). Asiala et al. (1996) reported that this type of encapsulation, namely the encapsulation of functions conceived of at the process level, is necessary to transition to the object level of function conception. Asiala et al. further reported that this type of encapsulation is mostly lacking in our experience with functions. Besides adhering to the GD and providing encapsulation opportunities, the IFI was also shown to create opportunities to address some prominent conceptual difficulties associated with the function concept, specifically 'what constitutes a function', 'univalence and injectivity' and 'multiple representations'. From the theoretical evaluation, it was concluded that the IFI is theoretically sound with respect to the design principles used in this study.

Part 2 of addressing the research question entailed the implementation of the IFI in a classroom setting, with subsequent qualitative analysis of a questionnaire given to participants. The aim was to determine if the IFI could be viable by looking for indications that participants experienced any broadening of their thinking with respect to functions, thus broadening their concept images. The IFI was put to the test in a classroom setting with 27 first-year Calculus students. From the results of the analysis, we saw that at least some participants reported gaining new insight on inputs and outputs of functions and the inverses of functions. Furthermore, it seems safe to conclude that for at least some participants, their ideas concerning what constitutes a function have been broadened. However, actual improved understanding of the function concept cannot be independently verified as it can be based only on the participants' own reporting. This is partly the fault of the questions of the questionnaire. They were intended to be sufficiently inviting to lead participants to provide rich responses from which true improved understanding could be judged. This did not happen to a sufficient extent. Seemingly, the questions were formulated to be too closedended. On a positive note, we can conclude from the results that the IFI has the potential to enrich the concept image (Tall \& Vinner, 1981, p. 151). The concept image is closely related to the schema level of the APOS theory in the sense that it is the construct one will utilise when confronted with solving an actual problem (Dubinsky \& McDonald, 2001, p. 3). Concluding that the concept image of participants can be enriched is based on the many instances of participants making a new connection between functions and everyday or real life and losing some of the restrictiveness regarding what constitutes a function. This enriched concept image gives us sufficient reason to claim proof of principle, thus concluding that the IFI is viable.

The theoretical evaluation of the IFI, together with the proof of principle that was obtained, led to the conclusion that there is sufficient indication that the use of the IFI can have merit and is therefore worthwhile to explore further. Subsequent qualitative and quantitative research will aim to independently verify not only if the GD is a true predictor of the mental constructions of participants, but also if the IFI can actually manage to improve participants' understanding of the function concept.

\section{Acknowledgements Competing interests}

I declare that no competing interests exist.

\section{Authors' contributions}

I declare that I am the sole author of this article.

\section{Ethical consideration}

Ethical clearance for the research conveyed in this article was obtained via the university general/human research ethics committee (GHREC), reference number UFS-HSD2019/ 0006/1505.

\section{Funding information}

This research received no specific grant from any funding agency in the public, commercial or not-for-profit sectors.

\section{Data availability statement}

The data that support the findings of this study are available from the corresponding author, upon reasonable request.

\section{Disclaimer}

The views and opinions expressed in this article are those of the author and do not necessarily reflect the official policy or position of any affiliated agency of the author. 


\section{References}

Akkoç, H., \& Tall, D. (2005). A mismatch between curriculum design and student learning: The case of the function concept. In D. Hewitt \& A. Noyes (Eds.), Proceedings of the sixth British Congress of Mathematics Education (pp. 1-8), University of Warwick, 30 March-02 April 2005.

Arnon, I., Cottrill, J., Dubinsky, E., Oktaç, A., Fuentes, S.R., Trigueros, M., \& Weller, K. (2014). APOS theory: A framework for research and curriculum development in mathematics education. New York, NY: Springer.

Asiala, M., Brown, A., De Vries, D.J., Dubinsky, E., Mathews, D., \& Thomas, K. (1996). A framework for research and curriculum development in undergraduate mathematics education in J. Kaput, A.H. Schoenfeld \& E. Dubinsky (Eds.), Research mathematics education in J. Kaput, A.H. Schoenfeld \& E. Dubinsky (Eds.), Research (vol. 6, pp. 1-32). Providence, RI: American Mathematical Society.

Ayers, T., Davis, G., Dubinsky, E., \& Lewin, P. (1988). Computer experiences in learning composition of functions. Journal for Research in Mathematics Education, 19(3), 246-259. https://doi.org/10.2307/749068

Bakar, M., \& Tall, D. (1991). Students' mental prototypes for functions and graphs. International Journal of Mathematical Education in Science and Technology, 23(1) 39-50. https://doi.org/10.1080/0020739920230105

Bakker, A., Smit, J., \& Wegerif, R. (2015). Scaffolding and dialogic teaching in mathematics education: Introduction and review. ZDM Mathematics Education 47(7), 1047-1065. https://doi.org/10.1007/s11858-015-0738-8

Bansilal, S., Brijlall, D., \& Trigueros, M. (2017). An APOS study on pre-service teachers' understanding of injections and surjections. The Journal of Mathematical
Behavior, 48, 22-37. https://doi.org/10.1016/j.jmathb.2017.08.002

Breidenbach, D., Dubinsky, E., Hawks, J., \& Nichols, D. (1992). Development of the process conception of function. Educational Studies in Mathematics, 23(3), 247-285. https://doi.org/10.1007/BF02309532

Carlson, M., Jacobs, S., Coe, E., Larsen, S., \& Hsu, E. (2002). Applying covariational reasoning while modeling dynamic events: A framework and a study. Journal for Research in Mathematics Education, 33(5), 352-378. https://doi.org/10.2307/ 4149958

Carlson, M., \& Oehrtman, M. (2005). Key aspects of knowing and learning the concep of function. Retrieved from http://www.maa.org/t and I/sampler/rs $9 . \mathrm{htm}$

Chimhande, T., Naidoo, A., \& Stols, G. (2017). An analysis of grade 11 learners' levels of understanding of functions in terms of APOS theory. Africa Education Review, 14(3-4), 1-19. https://doi.org/10.1080/18146627.2016.1224562

Confrey, J., \& Smith, E. (1991). A framework for functions: Prototypes, multiple representations and transformations. North American Chapter of the International representations and transformations. North American Chapter of the International
Group for the Psychology of Mathematics Education, Proceedings of the Annual Meeting, Blacksburg, VA, 13 October 1991 (pp. 57-63).

Donovan, M.S., \& Bransford, J.D. (Eds.). (2005). How students learn: Mathematics in the classroom. National Research Council Committee on How people learn. A targeted report for teachers, Division of behavioral and social sciences education. Washington, DC: The National Academies Press.

Doorman, M., Drijvers, P., Gravemeijer, K., Boon, P., \& Reed, H. (2012). Tool use and the development of the function concept: From repeated calculations to functional thinking. International Journal of Science and Mathematics Education 10, 1243-1267. https://doi.org/10.1007/s10763-012-9329-0

Dubinsky, E. (1991). Reflective abstraction in advanced mathematical thinking. In D.O. Tall (Ed.), Advanced mathematical thinking (pp. 95-123). Dordrecht: Kluwer.

Dubinsky, E. (2000). Using a theory of learning in college mathematics courses. In R.P. Burn (Ed.) Teaching and learning undergraduate mathematics (TALUM) Newsletter, 12. Leicester: The Mathematical Association (UK).

Dubinsky, E., \& McDonald, M.A. (2001). APOS: A constructivist theory of learning in undergraduate mathematics education research. In D. Holton (Ed.). The teaching and learning of mathematics at university level (pp. 275-282). Dordrecht: Kluwer academic Publishers.

Dubinsky, E., \& Wilson, R.T. (2013). High school students' understanding of the function concept. The Journal of Mathematical Behavior, 32(1), 83-101. https:// doi.org/10.1016/j.jmathb.2012.12.001

Eggleton, P. (1992). Motivation: A key to effective teaching. The Mathematics Educator, 3(2), 12 pages.

Gonzalez, R.C., \& Woods, R.E. (2017). Digital image processing (4th edn.). New York, NY: Pearson.
Harel, G., \& Dubinsky, E. (1992). The concept of function: Aspects of epistemology and pedagogy. Washington, DC: Mathematical Association of America.

Hoover, M., Mosvold, R., Ball, D.L., \& Lai, Y. (2016). Making progress on mathematical knowledge for teaching. The Mathematics Enthusiast, 13(1), 3-34.

Maharaj, A. (2010). An APOS analysis of students' understanding of the concept of a limit of a function. Pythagoras, 71, 41-52. https://doi.org/10.4102/pythagoras. v0i71.6

Maharajh, N., Brijlall, D., \& Govender, N. (2008). Preservice mathematics students notions of the concept definition of continuity in calculus through collaborative instructional design worksheets. African Journal of Research in Mathematics, Science and Technology Education, 12(suppl. 1), 93-106. https://doi.org/10.1080 10288457.2008.10740644

Makonye, J.P. (2014). Teaching functions using a realistic mathematics education approach: A theoretical perspective. International Journal of Science Education 7(3), 653-662. https://doi.org/10.1080/09751122.2014.11890228

Maree, K. (2016). First steps in research (2nd edn.) Pretoria: Van Schaik Publishers.

O'Shea, A., Breen, S., \& Jaworski, B. (2016). The development of a function concept inventory. International Journal of Research in Undergraduate Mathematics Education, 2, 279-296. https://doi.org/10.1007/s40753-016-0030-5

Pressley, M., Graham, S., \& Harris, K. (2006). The state of educational intervention research as viewed through the lens of literacy intervention. British Journal of Educational Psychology, 76(1), 1-19. https://doi.org/10.1348/000709905X66035

Reed, B. (2007). The effects of studying the history of the concept of function on student understanding of the concept. Unpublished Doctoral Dissertation. Kent, $\mathrm{OH}$ : Kent State University.

Sajka, M. (2003). A secondary school student's understanding of the concept of function: A case study. Educational Studies in Mathematics, 53(3), 229-254. https://doi.org/10.1023/A:1026033415747

Salgado, H., \& Trigueros, M. (2015). Teaching eigenvalues and eigenvectors using models and APOS theory. The Journal of Mathematical Behavior, 39, 100-20. https://doi.org/10.1016/j.jmathb.2015.06.005

Shrivakshan, G.T., \& Chandrasekar, A. (2012). A comparison of various edge detection techniques used in image processing, International Journal of Computer Science Issues, 9(1), 269-276.

Sierpinska, A. (1992). On understanding the notion of function. In G. Harel \& E. Dubinsky (Eds.), The concept of function: Aspects of epistemology and pedagogy (pp. 25-58). Washington, DC: The Mathematical Association of America.

Son, J., \& Hu, Q. (2015). The initial treatment of the concept of function in the selected secondary school mathematics textbooks in the US and China, International Journal of Mathematical Education in Science and Technology, 47(4), 505-530. https://doi.org/10.1080/0020739X.2015.1088084

Stewart, J. (2015). Single variable calculus (8th edn.). Boston, MA: Cengage Learning.

Tall, D., McGowen, M., \& DeMarois, P. (2000). The function machine as a cognitive root for the function concept. Paper presented at the Annual Meeting of the North American Chapter of the International Group for the Psychology of Mathematics Education (22nd, pp. 247-254, Tucson, AZ, October 7-10, 2000).

Tall, D., \& Vinner, S. (1981). Concept image and concept definition in mathematics with particular reference to limits and continuity. Educational Studies in Mathematics, 12, 151-169. https://doi.org/10.1007/BF00305619

Thompson, P.W. (1994). Students, functions, and the undergraduate curriculum. In E. Dubinsky, A.H. Schoenfeld, \& J.J. Kaput (Eds.), Research in collegiate mathematics education (pp. 21-44). Providence, RI: American Mathematical Society.

Vinner, S., \& Dreyfus, T. (1989). Images and definitions for the concept of function. Journal for Research in Mathematics Education, 20(4), 356-366. https://doi. org $/ 10.2307 / 749441$

Wadsworth, B.J. (1978). Piaget for the classroom teacher. New York, NY: Longman Inc.

Weller, K., Clark, J.M., Dubinsky, E., Loch, S., McDonald, M.A., \& Merkovsky, R. (2003). Student performance and attitudes in courses based on APOS theory and the ACE teaching cycle, in A. Selden, E. Dubinsky, G. Harel \& F. Hitt (Eds.), Research in Collegiate Mathematics Education V, CBMS issues in mathematics education (vol. 12, pp. 97-131). Providence, RI: American Mathematical Society.

Worley, P. (2015). Open thinking, closed questioning: Two kinds of open and closed question, Journal of Philosophy in Schools, 2(2), 17-29. https://doi.org/10.21913/ JPS.v2i2.1269 